\title{
The Schott Energy and the Reactive Energy in Electromagnetic Radiation and Mutual Couplings
}

This paper was downloaded from TechRxiv (https://www.techrxiv.org).

\section{LICENSE}

CC BY 4.0

SUBMISSION DATE / POSTED DATE

$01-11-2021 / 05-11-2021$

CITATION

xiao, gaobiao (2021): The Schott Energy and the Reactive Energy in Electromagnetic Radiation and Mutual Couplings. TechRxiv. Preprint. https://doi.org/10.36227/techrxiv.16914235.v1

$\mathrm{DOI}$

10.36227/techrxiv.16914235.v1 


\title{
The Schott Energy and the Reactive Energy in Electromagnetic Radiation and Mutual Couplings
}

\author{
Gaobiao Xiao, Shanghai Jiao Tong University
}

\begin{abstract}
In the proposed theory for analyzing the electromagnetic radiation and electromagnetic mutual couplings in vacuum, the electromagnetic energy associated with a source is basically separated into two parts. One is the Coulomb-velocity energy that is attached to the source, the other is the radiative energy that will depart from the source. A macroscopic Schott energy term is introduced to account for the interchange between the Coulombvelocity energy and the radiative energy. It has shown that the Schott energy will disappear with its sources but not simultaneously. It continues to exist for a short while after its sources disappeared and it will finally convert to radiative energy. The reactive electromagnetic energy consists of the Coulomb-velocity energy and the Schott energy, and the radiative energy also includes the contribution of the Schott energy. The theory provides a consistent formulation for the reactive energies and power balance equation both in time domain and frequency domain, which is validated with the radiation of a Hertzian dipole. By applying the Lienard-Wiechert potentials, this paper illustrates that the macroscopic Schott energy exactly agrees with the one obtained by many researchers for a nonrelativistic moving charged particle.
\end{abstract}

Index Terms - Schott energy, reactive energy, radiative energy, Lienard-Wiechert potential, electromagnetic coupling

\section{INTRODUCTION}

As observed by many researchers, although the classical electromagnetic theory has achieved tremendous success, some issues are still not solved satisfactorily, or have to be explained with quantum electromagnetic theory [1]-[6], such as the $4 / 3$ factor problem [2], the physical meaning of the vector potential, the violation of equivalence between mass and energy of an electron [1][5], and the electromagnetic radiation and coupling problem. All these issues have become century old problems, not only because they are quite complicated but also because they are probably considered as small flaws to the classical electromagnetic theory and have limited impact on engineering applications so far. However, these issues are difficulties associated directly with the classical electromagnetic theory. It is still of great significance to find proper solutions or interpretation within the frame of the classical electromagnetic theory, especially the issue concerning with the electromagnetic radiation and electromagnetic mutual couplings. There is still no widely accepted formulation for evaluating the stored reactive energies and Q factors of radiators [7]-[15]. The main difficulty may come from the fact that there is no clear definition in macroscopic electromagnetic theory for the reactive electromagnetic energy.

We have tried to provide a theory for the interpretation of the electromagnetic radiation and coupling based on the macroscopic Maxwell theory[16]-[20]. In the theory, the energy associated with a source in vacuum is separated into two parts, one accounts for the contribution from the Coulomb fields and the velocity fields, and is termed as Coulomb-velocity energy hereafter; the other is the radiative energy accounting for the contribution from the radiative fields. The Coulombvelocity energy is attached to its sources. It appears and disappears simultaneously with its sources. The radiative energy can leave its sources and propagate to infinity. It continues to exist after its sources disappeared. However, it can interact with other sources they encountered in the way and exchange energies with them by affecting their movement. Following the concept in the charged particle theory[21]-[25], a macroscopic Schott energy, $W_{D A}(t)$, is introduced in the proposed theory[20] to account for the energy exchanging between the Coulomb-velocity energy and the radiative energy, which is explicitly defined as a integral over the whole space,

$$
W_{D A}(t)=\int_{V_{\infty}} \frac{1}{2} \frac{\partial}{\partial t}[\mathbf{D}(\mathbf{r}, t) \cdot \mathbf{A}(\mathbf{r}, t)] d \mathbf{r}
$$

where $\mathbf{D}(\mathbf{r}, t)$ is the electric flux density and $\mathbf{A}(\mathbf{r}, t)$ the vector potential. They are from the same source in radiation problems, and from different sources in mutual coupling problems.

The Coulomb-velocity energy is denoted by $W_{\rho J}(t)$. It is the volume integral of the source-potential products over the source region $V_{s}$,

$$
W_{\rho J}(t)=\int_{V_{s}}\left[\frac{1}{2} \mathbf{J}(\mathbf{r}, t) \cdot \mathbf{A}(\mathbf{r}, t)+\frac{1}{2} \rho(\mathbf{r}, t) \phi(\mathbf{r}, t)\right] d \mathbf{r}
$$

The Coulomb-velocity energy includes the contribution from the Coulomb field and the velocity field, and is attached to the charge distribution $\rho(\mathbf{r}, t)$ and the current distribution $\mathbf{J}(\mathbf{r}, t)$. The scalar potential $\phi(\mathbf{r}, t)$ and the vector potential $\mathbf{A}(\mathbf{r}, t)$ evaluated at the observation point $\mathbf{r}$ and the time $t$ are defined in their usual way. They are subject to the Lorentz 
Gauge, and their reference zero points are put at the infinity. As clearly indicated in (2), $W_{\rho J}(t)$ equals zero if the sources vanished even if the potentials are not zero.

For the sake of convenience, a principal radiative energy is introduced in [20] as

$$
W_{\text {rad } 0}(t)=\int_{V_{\infty}} \frac{1}{2}\left(\frac{\partial \mathbf{D}}{\partial t} \cdot \mathbf{A}-\mathbf{D} \cdot \frac{\partial \mathbf{A}}{\partial t}\right) d \mathbf{r}
$$

It has been derived in [20] that the total electromagnetic energy associated with an electromagnetic source is the sum of the Coulomb-velocity energy and the principal radiative energy, i.e.,

$$
W_{\text {tot }}(t)=W_{\text {rad } 0}(t)+W_{\rho J}(t)
$$

The total reactive energy and the total radiative energy have to include the contribution of the Schott energy. They are expressed by

$$
\begin{aligned}
& W_{\text {react }}(t)=W_{\rho J}(t)+W_{D A}(t) \\
& W_{\text {rad }}(t)=W_{\text {rad } 0}(t)-W_{D A}(t)
\end{aligned}
$$

Therefore, the Schott energy plays the role of a bridge between the reactive energy and the radiative energy.

\section{Schott Energy for a Charged Particle}

Consider a nonrelativistic charged particle with charge $e$ and velocity $\mathbf{v}(t)$ in free space. The field generated by the charge can be derived from the following Lienard-Wiechert potentials [2][3],

$$
\begin{aligned}
& \phi(\mathbf{r}, t)=\frac{1}{4 \pi \varepsilon_{0}}\left[\frac{c e}{R c-\mathbf{R} \cdot \mathbf{v}}\right]_{t^{\prime}}=\frac{e}{4 \pi \varepsilon_{0}}\left[\frac{1}{R(1-\mathbf{n} \cdot \boldsymbol{\beta})}\right]_{t^{\prime}} \\
& \mathbf{A}(\mathbf{r}, t)=\frac{\mu_{0}}{4 \pi}\left[\frac{e c \mathbf{v}}{R c-\mathbf{R} \cdot \mathbf{v}}\right]_{t^{\prime}}=\frac{e}{4 \pi \varepsilon_{0} c}\left[\frac{\boldsymbol{\beta}}{R(1-\mathbf{n} \cdot \boldsymbol{\beta})}\right]_{t^{\prime}}
\end{aligned}
$$

where $\mathbf{R}=\mathbf{r}-\mathbf{x}\left(t^{\prime}\right), R=\left|\mathbf{r}-\mathbf{x}\left(t^{\prime}\right)\right|$, and $\mathbf{x}\left(t^{\prime}\right)$ is the trajectory of the moving charge. $t^{\prime}=t-R / c$ is the retarded time, and $c$ is the light velocity in vacuum. Note that the quantities at the righthand side of (7) and (8) are evaluated at $t^{\prime}$. $\mathbf{v}\left(t^{\prime}\right)=d \mathbf{x}\left(t^{\prime}\right) d t^{\prime}, \quad \boldsymbol{\beta}=\mathbf{v} / c$, and $\mathbf{n}=\mathbf{R} / R$.

The non-relativistic Schott energy of the moving charge is found to be (eq. (16) in [23], eq. (4) in [24], eq.(3.24) in [25])

$$
E_{S}(t)=-\frac{1}{4 \pi \varepsilon_{0}} \frac{2 e^{2}}{3 c^{3}} \mathbf{a} \cdot \mathbf{v}
$$

where $\mathbf{a}$ is the acceleration of the charge. Note that there is a factor $1 /\left(4 \pi \varepsilon_{0}\right)$ because SI unit system is used in this paper while the results in some references were obtained in Gaussian (cgs) system of units.

We are now to show that $E_{s}(t)$ can be derived by applying the Lienard-Wiechert potentials and the corresponding fields to $W_{D A}(t)$. Recalling that $\mathbf{E}=-\nabla \phi-\partial \mathbf{A} / \partial t$, and making use of the Lorentz Gauge $\nabla \cdot \mathbf{A}+c^{-2} \partial \phi / \partial t=0$, we can transform $W_{D A}(t)$ to an integral of the potentials,

$$
W_{D A}(t)=-\frac{\varepsilon_{0}}{4} \frac{\partial^{2}}{\partial t^{2}} \int_{V_{\infty}}\left[c^{-2} \phi^{2}(\mathbf{r}, t)+\mathbf{A}(\mathbf{r}, t) \cdot \mathbf{A}(\mathbf{r}, t)\right] d \mathbf{r}
$$

Since (10) is a total time derivative, we can approximately evaluate the integration in a simple way. For $v=|\mathbf{v}| \ll c$, the potential terms can be expanded with power series of $(\mathbf{n} \cdot \boldsymbol{\beta})$. Keeping the terms to the second order we have

$$
\begin{gathered}
\phi^{2}(\mathbf{r}, t) \approx\left(\frac{e}{4 \pi \varepsilon_{0}}\right)^{2}\left[\frac{1}{R^{2}}\left(1+2 \mathbf{n} \cdot \boldsymbol{\beta}+(\mathbf{n} \cdot \boldsymbol{\beta})^{2}\right)\right]_{t^{\prime}} \\
\mathbf{A} \cdot \mathbf{A} \approx\left(\frac{e}{4 \pi \varepsilon_{0} c}\right)^{2}\left[\frac{\boldsymbol{\beta} \cdot \boldsymbol{\beta}}{R^{2}}\right]_{t^{\prime}}
\end{gathered}
$$


Assume that the source exists in a very short time period of $\left[t^{\prime}, t^{\prime}+d t\right]$, and $t^{\prime}=t-r_{o} / c$. Here $r_{o}$ is a very small distance from the charged particle at $\mathbf{x}\left(t^{\prime}\right)$. In can be checked that, except the first term in the righthand side of (11), all other terms in the integrand are approximately nonzero only in a spherical shell with radius $r_{o}$ to $r_{o}+c d t$. In the spherical coordinate system with origin locating at $\mathbf{x}\left(t^{\prime}\right)$, we have $R=r$. The integral of (10), except the first term in the right hand of (11), can be cast into

$$
\begin{aligned}
d I_{A \phi}(t) & =d \int_{V_{\infty}}\left[c^{-2} \phi^{2}(\mathbf{r}, t)+\mathbf{A}(\mathbf{r}, t) \cdot \mathbf{A}(\mathbf{r}, t)\right] \\
& \approx\left(\frac{e}{4 \pi \varepsilon_{0} c}\right)^{2} \int_{0}^{2 \pi} \int_{0}^{\pi} \int_{r_{o}}^{r_{0}+c d t}\left\{\frac{1}{r^{2}}\left[\left(2 \mathbf{n} \cdot \boldsymbol{\beta}+(\mathbf{n} \cdot \boldsymbol{\beta})^{2}+\boldsymbol{\beta} \cdot \boldsymbol{\beta}\right)\right]\right\} r^{2} \sin \theta d r d \theta d \varphi \\
& \approx\left\{\left(\frac{e}{4 \pi \varepsilon_{0} c}\right)^{2} \int_{0}^{2 \pi} \int_{0}^{\pi}\left[\left(2 \mathbf{n} \cdot \boldsymbol{\beta}+(\mathbf{n} \cdot \boldsymbol{\beta})^{2}+\boldsymbol{\beta} \cdot \boldsymbol{\beta}\right)\right] \sin \theta d \theta d \varphi\right\} c d t
\end{aligned}
$$

A convenient choice is to put $\boldsymbol{\beta}$ on the z-axis, then $\mathbf{n} \bullet \boldsymbol{\beta}=|\boldsymbol{\beta}| \cos \theta$ and the integrand is symmetrical with z-axis. Note that $\int_{0}^{\pi} \cos \theta \sin \theta d \theta=0$, and $\int_{0}^{\pi} \cos ^{2} \theta \sin \theta d \theta=2 / 3$. By carrying out the integration and putting $d t$ to the left side of the equation we have

$$
\frac{d I_{A \phi}(t)}{d t}=\frac{1}{4 \pi \varepsilon_{0}} \frac{4 e^{2}}{3 \varepsilon_{0} c} \boldsymbol{\beta} \cdot \boldsymbol{\beta}=\frac{1}{4 \pi \varepsilon_{0}} \frac{4 e^{2}}{3 \varepsilon_{0} c^{3}} \mathbf{v} \cdot \mathbf{v}
$$

It can be checked straightforwardly that the integration of the first term in the righthand side of (11) is a constant, which will vanish after differentiate with respect to time $t$. Therefore, it can be deduced from (14) that

$$
W_{D A}(t)=-\frac{\varepsilon_{0}}{4} \frac{d}{d t}\left(\frac{d I_{A \phi}(t)}{d t}\right) \approx-\frac{1}{4 \pi \varepsilon_{0}} \frac{2 e^{2}}{3 c^{3}} \mathbf{a} \cdot \mathbf{v}
$$

which is exactly in agreement with (9).

\section{CONCLUSIONS}

The electromagnetic radiation and mutual couplings are fundamental classical electromagnetic problems. The author believe that it is meaningful to handle the issue in the frame of the classical electromagnetic theory, and it is not always necessary to resort to quantum electromagnetic theory. The theory proposed in [20] is aimed to provide a more compatible interpretation for the electromagnetic radiation and mutual couplings in the language of macroscopic Maxwell theory so as that those commonly used quantities like the reactive electromagnetic energies, radiative energies, Q-factors, and the electromagnetic coupling energies can be evaluated in a consistent manner. The introduction of the Schott energy in the macroscopic electromagnetic radiation/coupling formulation perhaps can make it more intuitive to understand the process of building the energy balance in a typical electromagnetic radiation or mutual coupling system. In the case of the Hertzian dipole, the reactive energies obtained with the proposed theory exactly agree with those predicted by using the equivalence circuit model [26]. To the best of my knowledge, no other related formulation can get such verification. This note shows that the macroscopic Schott energy is indeed in agreement with the one introduced in charged particle theory one century ago. Although the significance of the Schott energy itself still has not been fully clarified in charged particle theory, the author still wants to try to convince the readers that the role of the Schott energy in electromagnetic radiation and mutual couplings has to be taken into account.

\section{References}

[1] J. Kalckar, J. Lindhard, O. Ulfbeck, "Self-mass and equivalence in special relativity," Mat.-Fys. Medd. Danske Vid. Selsk., 40:11, 1982.

[2] J. D. Jackson, "Classical Electrodynamics.” 3rd ed., John Wiley \& Sons: New York, NY, USA, 1998.

[3] F. Rohrlich, "Classical Charged Particles," 3rd ed., World Scientific Publishing: Singapore, 2007.

[4] A. K. Singal, "Compatibility of Larmor's formula with radiation reaction for an accelerated charge," Found. Phys., vol. 46, 2016.

[5] M. Kh. Khokonov, J. U. Andersen, "Equivalence between self-energy and self-mass in classical electron model," Found. Phys., vol. 49, pp. 750-782, 2019.

[6] T. W. Barrett, "Electromagnetic phenomena not explained by maxwell's equations." In book: Essays on the Formal Aspects of Electromagnetic Theory, pp. 6-86, Jun. 1993.

[7] R. E. Collin and S. Rothschild, "Evaluation of antenna Q," IEEE Trans. Antennas Propag., vol. 12, no. 1, pp. 23-27, Jan. 1964.

[8] A. Shlivinski and E. Heyman, "Time-domain near-field analysis of short pulse antennas-Part I: Spherical wave (multipole) expansion," IEEE Trans. Antennas Propag., vol. 47, no. 2, pp. 271-279, Feb. 1999.

[9] A. Shlivinski and E. Heyman, "Time-domain near-field analysis of short pulse antennas—Part II: Reactive energy and the antenna Q," IEEE Trans. Antennas Propag., vol. 47, no. 2, pp. 280-286, Feb. 1999. 
[10] A. D. Yaghjian, "Internal energy, Q-energy, Poynting's theorem, and the stress dyadic in dispersive material," IEEE Trans. Antennas Propag., vol. 55, no. 6, pp. 1495-1505, Jun. 2007.

[11] G. A. E. Vandenbosch, "Reactive energies, impedance, and Q factor of radiating structures," IEEE Trans. Antennas Propag., vol. 58, no. 4, pp. 1112-1127, Apr. 2010.

[12] G. A. E. Vandenbosch, "Radiators in time domain-Part I: Electric, magnetic, and radiated energies," IEEE Trans. Antennas Propag., vol. 61, no. 8, pp. 3995-4003, Aug. 2013.

[13] G. A. E. Vandenbosch, "Radiators in time domain-Part II: Finite pulses, sinusoidal regime and Q factor," IEEE Trans. Antennas Propag., vol. 61, no. 8, pp. 4004-4012, Aug. 2013.

[14] M. Gustafsson and B. L. G. Jonsson, "Antenna Q and stored energy expressed in the fields, currents, and input impedance," IEEE Trans. Antennas Propag., vol. 63, no. 1, pp. 240-249, Jan. 2015.

[15] G. A. E. Vandenbosch, "Recoverable energy of radiating structures," IEEE Trans. Antennas Propag., vol. 65, no. 7, pp. 3575-3588, Jul. 2017.

[16] G. B. Xiao, C. Xiong, S. Huang, R. Liu, Y. Hu, "A new perspective on the reactive electromagnetic energies and Q factors of antennas," IEEE Access, vol. 8, 8999565, pp. 173790-173803, Oct. 2020.

[17] G. B. Xiao, "Electromagnetic energy balance equations and Poynting Theorem," TechRxiv. Preprint. https://doi.org/ 10.36227/ techrxiv.12555698.v1, 2020.

[18] G. B. Xiao, Y. Hu and S. Xiang, "Comparison of five formulations for evaluating Q factors of antennas," 2020 IEEE MTT-S International Conference on Numerical Electromagnetic and Multiphysics Modeling and Optimization (NEMO). IEEE, 2020.

[19] G. B. Xiao, “A theory for analysis of pulse electromagnetic radiation," TechRxiv. Preprint. https://doi.org/10.36227/techrxiv. 14054051.v4, 2021.

[20] G. B. Xiao, “A theory for electromagnetic radiation and coupling.” TechRxiv. Preprint. https://doi.org/10.36227/techrxiv.16686112. v2, 2021

[21] G. A. Schott, "Electromagnetic radiation and the mechanical reactions arising from it," Cambridge University Press: Cambridge, UK, 1912.

[22] T. Nakamura, "On the Schott term in the Lorentz-Abraham-Dirac equation," Quantum Beam Sci., vol. 4, pp. 34, 2020.

[23] Ø. Grøn, "The significance of the Schott energy for energy-momentum conservation of a radiating charge obeying the LorentzAbraham-Dirac equation," Am. J. Phys., vol. 79, no. 1, pp. 115-122, 2011.

[24] D. R. Rowland, "Physical interpretation of the Schott energy of an accelerating point charge and the question of whether a uniformly accelerating charge radiates," Eur. J. Phys., vol. pp. 31, 1037-1051, Jul. 2010.

[25] E. Eriksen, Ø. Grøn, "Electrodynamics of hyperbolically accelerated charges: IV. Energy-momentum conservation of radiating charged particles," Annals of Physics, 297, pp. 243-294, 2002.

[26] L. J. Chu, "Physical limitations on omni-directional antennas," J. Appl. Phys., vol. 19, no. 12, pp. 1163-1175, 1948. 